\title{
Phenotypic Diversity of Photosynthetic Characteristics in 16 Cucumber Accessions Selected from the Core Collection
}

\author{
Yuan-Yuan ZHAO, Sha LI, Rui-Jin ZHANG, Li-Hong SU, \\ Huan-Xiu LI, Yun-Song LAI ${ }^{a}$, \\ Institute of Pomology and Olericulture, Sichuan Agricultural University, Chengdu \\ 611130, P.R.China \\ aemail: laiyunsong@126.com \\ ${ }^{*}$ Corresponding author
}

Keywords: Cucumber, Photosynthetic characteristics, Light response curves, Phenotypic variation.

\begin{abstract}
To investigate the phenotypic variation of cucumber photosynthesis, net photosynthetic efficiency of cucumbers were measured by LI-6400XT Portable Photosynthesis System. Non-rectangular hyperbolic model was adopted to fit the light response curve. We used 16 cucumber accessions selected from the core collection that consists of over 400 accessions. As a result, cucumbers show a moderate variance in the phenotype of light compensation point $(L C P)$, light saturation point $(L S P)$ and apparent quantum efficiency $(A Q Y)$, dark respiration rate $\left(R_{d}\right)$, and maximum net photosynthetic rate $\left(A_{\max }\right)$. In a detail, the coefficient of variation $(\mathrm{CV})$ of $L C P$ and $R_{d}$ is $48.97 \%$ and $48.09 \%$, respectively, which is relatively high. In contrast, the CV values of $A_{\max }, L S P$ and $A Q Y$ are all smaller than $25 \%$. These results help us get better understanding about the phenotypic variation of photosynthesis in cucumber germplasm.
\end{abstract}

\section{Introduction}

The cucumber (Cucumis sativus L.) is an important vegetable in the world whose planting area is only the second to tomato [1]. A core collection of cucumber germplasm consisting of 473 accessions was established previously based on the genetic diversity study using over 4000 original collections from all over the world [2, $3]$. Photosynthesis converts light energy into chemical energy and therefore is the bases of crop yield. Generally, dry matter weight ascribed to photosynthesis takes $90 \%-95 \%$, while that ascribed to root absorption takes only 5\% to $10 \%$. There is a positive correlation between photosynthetic rate and the plant dry matter accumulation ability [12]. Lots of studies have already demonstrated that light intensity and quality intensively affect the photosynthesis reaction and subsequently the plant growth rate [4].To our best knowledge, few studies revealed the photosynthetic characteristics in cucumber germplasm so far. In this study, we investigated the photosynthetic traits of 16 cucumber accessions and thereafter calculated light compensation point $(L C P)$, light saturation point $(L S P)$, the apparent quantum efficiency $(A Q Y)$, dark respiration rate $\left(R_{d}\right)$, and maximum net photosynthetic rate $\left(A_{\max }\right)$.

\section{Materials and Methods}

\section{Plant Materials}

A total of 16 cucumber accessions were selected from the core collection to perform the assay. These accessions are from India, Russia, Spain, Germany, America, Japan, as well as provinces of Xinjiang, Guizhou, Fujian, Henan, Shandong, Guangxi, Anhui, 
Hubei, Sichuan, Yunnan in China. All the cucumber plants were grown in greenhouse at the same time in spring, 2015. The fifth top leaves of two month old plants were subjected to photosynthesis measurement.

\section{Light Response Curve Measurement}

Photosynthetic characteristics were measured with a Portable Photosynthesis System LI-6400 (LI-COR Inc., USA). All parameters were measured between 10:00 A.M. and 11:00 A.M. The light-response curves of $\mathrm{P}_{\mathrm{N}}$ with light intensity changing was made with the PAR setting values 1800, 1500, 1200, 1000, 800, 600, 400, 200, 150, 100, 75, $50,25,0\left(\mu \mathrm{mol} \cdot \mathrm{m}^{-2} \cdot \mathrm{s}^{-1}\right)$. The measurement was conducted under CO2 concentration of $400 \mu \mathrm{mol} \cdot \mathrm{mol}^{-1}$. Light response curve fitting was performed using non-rectangular hyperbolic model. The fitting formula was as follows [5]:

$$
A=\quad \frac{A \max \times P P D F}{\sqrt{(A Q Y \times P P D F+A m a x}}-R d
$$

$A_{\max }$, the maximum net photosynthetic efficiency. $A$, net photosynthetic rate. $A Q Y$, apparent quantum efficiency. $R_{d}$, dark respiration rate. $K$, the optical response curve angle. $P P D F$, photosynthetically active radiation.

Light response curves were fitted using an iterative method to calculate the apparent quantum efficiency $(A Q Y)$, dark respiration rate $\left(R_{d}\right)$, maximum net photosynthetic rate $\left(A_{\max }\right)$. The formula was as follows [6]:

$$
A=A Q Y * P P D F-R_{d}\left(P P D F<=200 \mu \mathrm{mol} \cdot \mathrm{m}^{-2} \cdot \mathrm{s}^{-1}\right)
$$

In this formula, light compensation point $(L C P)$ was calculated when $A$ is 0 ; light saturation point $(L S P)$ was calculated when $A$ is $A_{\max }$.

\section{Results and Discussion}

\section{Light Response Curve Analysis}

$\mathrm{P}_{\mathrm{N}}$ responses to PAR of the 16 cucumber species showed significant differences under the light intensity from 400 to $2400 \mu \mathrm{mol} \cdot \mathrm{m}^{-2} \cdot \mathrm{s}^{-1}$ (Fig. 1). $\mathrm{P}_{\mathrm{N}}$ increased rapidly when PAR increased from 0 to $800 \mu \mathrm{mol} \cdot \mathrm{m}^{-2} \cdot \mathrm{s}^{-1}$. Based on the light response curves, photosynthetic parameters ( $\left.L C P, L S P, A Q Y, R_{d}, A_{\max }\right)$ were also calculated (Table 1). The $A_{\max }$ value ranges $7.801-18.119 \mu \mathrm{mol} \cdot \mathrm{m}^{-2} \cdot \mathrm{s}^{-1}$. This value is lower to major cereal crops $e . g$. maize [7], rice [8] but comparable to most vegetables e.g. pepper [9], tomato [10]. $L C P$ and $L S P$ are used to reflect the ability of plants to use light in low and high intensity $[11,12]$.The $L S P$ value ranges from 237.40 to $647.67 \mu \mathrm{mol} \cdot \mathrm{m}^{-2} \cdot \mathrm{s}^{-1}$. The $L C P$ value ranges from 2.401 to $29.453 \mu \mathrm{mol} \cdot \mathrm{m}^{-2} \cdot \mathrm{s}^{-1}$ and the average number is 16.241 $\mu \mathrm{mol} \cdot \mathrm{m}^{-2} \cdot \mathrm{s}^{-1}$, which is significantly lower than that in many other plant species [13]. This suggests that cucumbers are capable of adapting to weak light. Based on these above results, strong light intensity is in fact not necessary for cucumber growth although cucumbers are known as heliophile. $A Q Y$ was calculated as the inclination of the initial slop of the light response curve of net photosynthesis. The value of $A Q Y$ reflects potential plant photosynthetic capacity and energy use efficiency [14, 15]. Cucumber $A Q Y$ value ranges $0.023-0.046$ which is higher than most plant species. Plants usually have an $A Q Y$ value of $0.03-0.05$. The $R_{d}$ value ranges $0.128-1.071$ $\mu \mathrm{mol} \cdot \mathrm{m}^{-2} \cdot \mathrm{s}^{-1}$. Lower $R_{d}$ value indicates higher ability to accumulate dry matters. However, many accessions that have higher photosynthetic rate like No. 16, No. 81, 
and No. 97 etc. also have higher $R_{d}$ value. These cucumbers have higher growth rate due to the higher rate of metabolism.

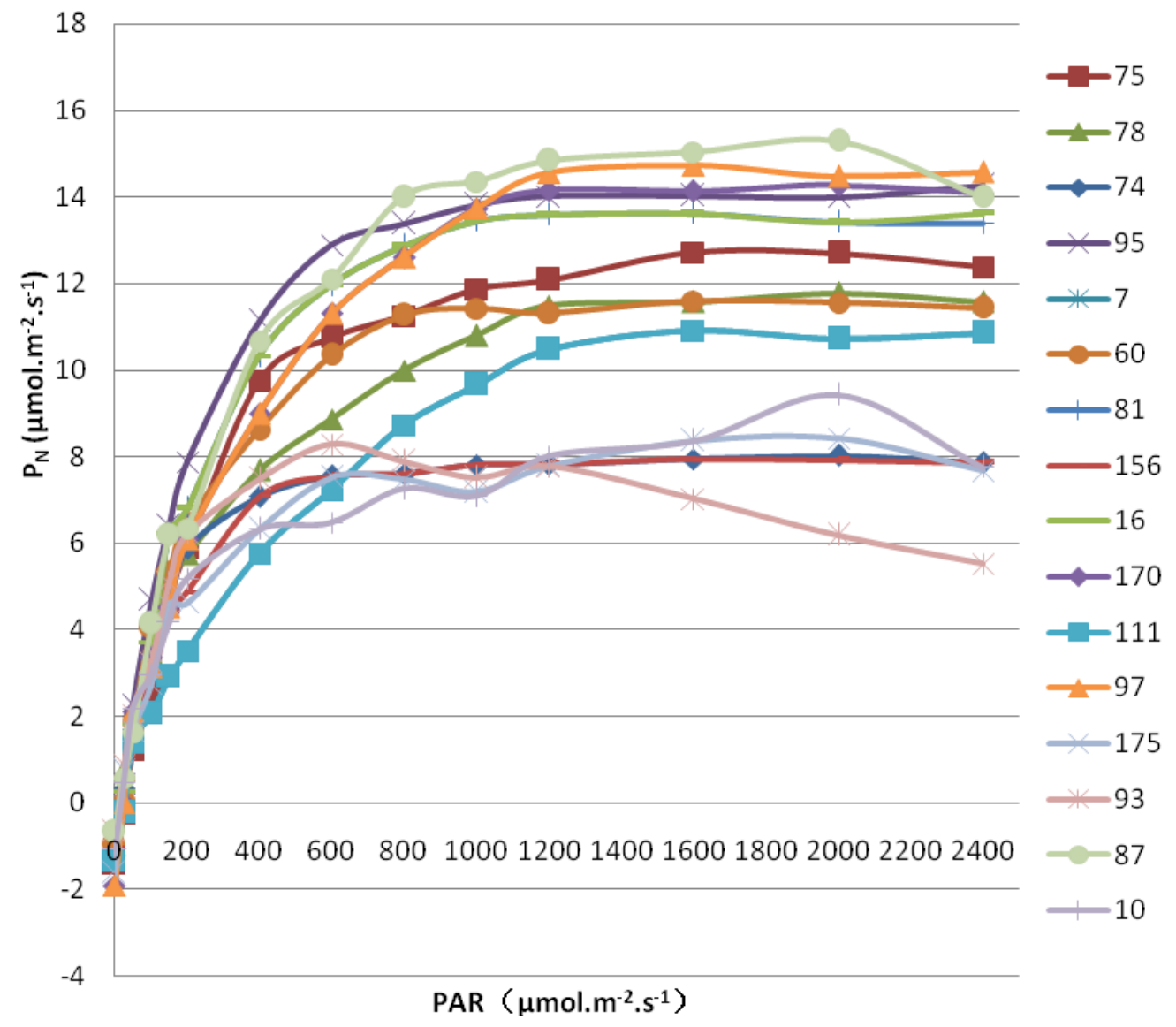

Figure 1. Responses of the net photosynthetic rate $\left(\mathrm{P}_{\mathrm{N}}\right)$ to photosynthetically active radiation (PAR) in the 16 cucumber accessions.

Table 1. Photosynthetic parameters of light-response curve

\begin{tabular}{|c|c|c|c|c|c|}
\hline $\begin{array}{c}\text { Accession } \\
\text { No }\end{array}$ & $\begin{array}{c}\text { LCP } \\
(\mu \mathrm{mol} \cdot \mathrm{m}-2 \cdot \mathrm{s}-1)\end{array}$ & $\begin{array}{c}\text { LSP } \\
(\mu \mathrm{mol} \cdot \mathrm{m}-2 \cdot \mathrm{s}-1)\end{array}$ & AQY & $\begin{array}{c}\text { Rd } \\
(\mu \mathrm{mol} \cdot \mathrm{m}-2 \cdot \mathrm{s}-1)\end{array}$ & $\begin{array}{c}\text { Amax } \\
(\mu \mathrm{mol} \cdot \mathrm{m}-2 \cdot \mathrm{s}-1)\end{array}$ \\
\hline 7 & 27.057 & 511.435 & 0.023 & 0.620 & 14.212 \\
16 & 15.537 & 393.530 & 0.041 & 0.637 & 15.498 \\
60 & 14.094 & 366.588 & 0.038 & 0.537 & 13.430 \\
81 & 19.639 & 390.138 & 0.042 & 0.827 & 15.589 \\
97 & 24.436 & 518.880 & 0.037 & 0.902 & 18.119 \\
111 & 27.057 & 647.668 & 0.023 & 0.620 & 14.212 \\
156 & 9.799 & 326.089 & 0.029 & 0.283 & 9.141 \\
170 & 23.524 & 494.768 & 0.037 & 0.870 & 17.436 \\
74 & 15.653 & 288.130 & 0.033 & 0.518 & 9.019 \\
75 & 29.453 & 425.854 & 0.036 & 1.071 & 14.429 \\
78 & 8.523 & 426.076 & 0.033 & 0.282 & 13.821 \\
95 & 13.340 & 368.991 & 0.046 & 0.608 & 16.143 \\
93 & 2.401 & 237.398 & 0.033 & 0.128 & 7.801 \\
87 & 6.624 & 457.851 & 0.038 & 0.248 & 16.921 \\
10 & 7.825 & 341.570 & 0.029 & 0.228 & 9.712 \\
175 & 14.895 & 355.575 & 0.029 & 0.438 & 10.016 \\
\hline
\end{tabular}

\section{Dispersion of Photosynthetic Parameters TRAITS}

Using SPSS software, traits dispersion of photosynthetic parameters were analyzed (Table 2). The dispersion rate of $L C P, L S P, A Q Y, R_{d}$, and $A_{\max }$ are $48.969 \%, 23.820 \%$, 
$17.647 \%, 48.094 \%$, and $23.766 \%$, respectively. These results indicate there is a big difference in the phenotypic variance of the photosynthesis parameters. Considering the assayed cucumber accessions are from different countries and represent a genetic diversity in cucumber germplasm, we suppose that cucumbers have evolved a big variance to adapt to week light condition $(L C P)$ but not strong light condition $(L S P)$. According to other reports, the coefficient of variation of major cucumber agronomic traits ranges $9 \%-88 \%$ [16].Generally speaking, there is a moderate variance in the phenotype of cucumber photosynthesis.

Table 2. Photosynthetic parameters of light-response curve

\begin{tabular}{|c|r|r|r|r|r|}
\hline & \multicolumn{1}{|c|}{$L C P$} & \multicolumn{1}{|c|}{$L S P$} & $A Q Y$ & \multicolumn{1}{c|}{$R_{\mathrm{d}}$} & $A_{\max }$ \\
\hline Average & 16.241 & 409.409 & 0.034 & 0.551 & 13.469 \\
SD & 7.953 & 97.523 & 0.006 & 0.265 & 3.201 \\
CV $(\%)$ & 48.969 & 23.820 & 17.647 & 48.094 & 23.766 \\
\hline
\end{tabular}

$\mathrm{SD}$, Standard deviation; $\mathrm{CV}$, coefficient of variation; $\mathrm{CV}=\mathrm{SD} /$ Average.

\section{Acknowledgement}

This work is supported by the Department of Education of Sichuan Province, China (Grant-In-Aid for Scientific Research No. 14ZA0014).

\section{References}

[1] J.E. Staub, M.D. Robbins and T.C. Wehner, Cucumber, in: J. Prohens, F. Nuez (Eds), Vegetables I., Springer, New York, 2008, pp. 241-282.

[2] Guangping Zhang, Xixiang Li, Changping Xiang, Di Shen, Wenling Wang and Jiangping Song. Studies on the methods of establishing cucumber core collection based on RAPD analysis. Acta Horticulturae Sinica, 33 (2006) 260-265. (in Chinese)

[3] Jianjian Qi, Xin Liu, Di Shen, Han Miao, Bingyan Xie, Xixiang Li, Peng Zeng, Shenhao Wang, Yi Shang, XingfangGu, Yongchen Du, Ying Li, Tao Lin, Jinhong Yuan,Xueyong Yang, Jinfeng Chen,Huiming Chen, XingyaoXiong, KeHuang, ZhangjunFei, Linyong Mao, Li Tian, Thomas Städler, Susanne S Renner, Sophien Kamoun et al. A genomic variation map provides insights into the genetic basis of cucumber domestication and diversity. Nature Genetics, 45 (2013) 1510-1515.

[4]M. Olaizola, O. D. Eirik. Effects of light intensity and quality on the growth rate and photosynthetic pigment content of Spirulina platensis. Journal of Applied Phycology, 2 (1990) 97-104.

[5] J.H.M. Thornley. Instantaneous canopy photosynthesis: Analytical expressions for sun and shade leaves based on exponential light decay down the canopy and an acclimated non-rectangular hyperbola for leaf photosynthesis. Annals of Botany, 89 (2002) 451-458.

[6] Peixi Su, Guodong Cheng, Qiaodi Yan and Xinmin Liu. Photosynthetic regulation of $\mathrm{C} 4$ desert plant Haloxylonammodendron under drought stress. Plant Growth Regulation, 51 (2007) 139-147. (in Chinese)

[7] Dongmei Li, Kuihua Zhao, Yanbo Wang, Hua Qi, DaweiWang, Yanan Wu, Haiyan Zhao, ChunlingZhang and MingLiu. Response of photosynthesis characters to 
low-light stress for maize hybrids withdifferent density tolerances. Journal of Maize Science, 21 (2013) 52-56. (in Chinese)

[8] L.-J. Ou, W.-J. Li, L.-F. Tian, Z. Chen, X.-J. Dai, L.-X. Deng, Y.-L. Qiu, H. Li, P. Liu, M.-Z. Liang, S. Luan and L.-B. Chen. Photosynthetic characteristics of C4 trait in chlorina mutant of rice (Oryza sativa L.). Photosynthetica, 46 (2008) 589-594.

[9] Ling Fu,XiaomeiBai,XianheYang,GuoxiuWu and XizhenAi.Effects of grafted on photosynthetic characteristics, yield and quality of pepper. ActaHorticulturaeSinica, 40 (2013) 449-457. (in Chinese)

[10] KailiChen,JianmingLi, HuiqiangHe, XiaohuiHu, YongzheYao andSanjieSun. Effects of water on photosynthesis in different age of tomato leaves. ActaEcologicaSinica, 33 (2013) 4919-4929. (in Chinese)

[11] LingduoBu,RenheZhang,Yu Chang,JiquanXue and MiaomiaoHan. Response of photosynthetic characteristics to water stress of maize leaf in seeding. ActaEcologicaSinica, 30 (2010) 1184-1192. (in Chinese)

[12] Daquan Xu. Photosynthetic Efficiency. Shanghai Scientific and Technical Publishers, Shanghai, 2002, pp. 821-834. (in Chinese)

[13] Yan Wang. Comparative studies on utilization light characteristics and shade tolerance of 14 ground covers. Journal of Zhejiang Forestry College, 22 (2005) 8-13. (in Chinese)

[14] XihuanZhang,Heming Xin,ChunyuTian and Baozhong Huang.Studied on photosynthetic characteristics of fuchengmumecultivar. ActaAgriculturaeBoreali-occidentalisSinica, 16 (2007) 198-201. (in Chinese)

[15] Kezhang Xu. Plant Physiology. China Agriculture Press, Beijing, (2007) 163-164. (in Chinese)

[16] Qiwei Cao, Zhifeng Wang, Weihua Zhang and Xiaolei Sun. Evaluation of different cucumber varieties in several precocious traits, Journal of Shandong Agricultural Sciences, 9 (2008) 16-18. (in Chinese) 